\title{
Mobile phone technologies improve adherence to antiretroviral treatment in a resource-limited setting: a randomized controlled trial of text message reminders
}

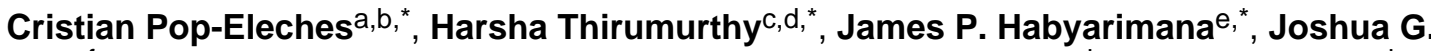 \\ Zivin $^{f}$, Markus P. Goldstein ${ }^{g}$, Damien de Walque ${ }^{g}$, Leslie MacKeen ${ }^{\mathrm{h}}$, Jessica Haberer ${ }^{\mathrm{i}, \mathrm{o}}$, \\ Sylvester Kimaiyo', John Sidle ${ }^{k, l}$, Duncan Ngare ${ }^{m}$, and David R. Bangsberg ${ }^{n, p}$
}

aSchool of International and Public Affairs bepartment of Economics, Columbia University, New York, New York 'Department of Health Policy and Management, Gillings School of Global Public Health, University of North Carolina at Chapel Hill, Chapel Hill, North Carolina dThe World Bank ePublic Policy Institute, Georgetown University, Washington, District of Columbia fSchool of International Relations and Pacific Studies, University of California, San Diego, California gDevelopment Research Group, The World Bank hBureau for Global Health, United States Agency for International Development, Washington, District of Columbia 'Department of Medicine, Massachusetts General Hospital, Boston, Massachusetts, USA iSchool of Medicine, Moi University, Eldoret, Kenya kDivision of General Internal Medicine, Indiana University School of Medicine, Indianapolis, Indiana, USA 'Department of Medicine, Faculty of Health Sciences mSchool of Public Health, Moi University, Eldoret, Kenya ${ }^{n}$ Ragon Institute of MGH, MIT and Harvard ${ }^{\circ}$ Massachusetts General Hospital Center for Global Health, Boston, Massachusetts, USA pMbarara University of Science and Technology, Mbarara, Uganda

\section{Abstract}

Objective-There is limited evidence on whether growing mobile phone availability in subSaharan Africa can be used to promote high adherence to antiretroviral therapy (ART). This study tested the efficacy of short message service (SMS) reminders on adherence to ART among patients attending a rural clinic in Kenya.

Design-A randomized controlled trial of four SMS reminder interventions with 48 weeks of follow-up.

Methods-Four hundred and thirty-one adult patients who had initiated ART within 3 months were enrolled and randomly assigned to a control group or one of the four intervention groups. Participants in the intervention groups received SMS reminders that were either short or long and

(C) 2011 Wolters Kluwer Health | Lippincott Williams \& Wilkins

Correspondence to Harsha Thirumurthy, PhD, The World Bank, 1818 H Street NW, Washington, DC 20433, USA. hthirumurthy@worldbank.org, harsha@unc.edu.

${ }^{*}$ C.P.-E., H.T. and J.P.H. contributed equally to the writing of this article.

C.P., H.T., J.P.H., J.G.Z., M.P.G., J.S., and D.N. designed the study. Data collection was performed by L.M. under the supervision of D.N., H.T., J.S., and J.P.H. Data were analyzed by C.P., H.T., J.P.H. and interpreted by C.P., H.T., J.P.H., and D.R.B. The manuscript was written by C.P., H.T., J.P.H., J.H., and D.R.B. and revision of the manuscript was performed by D.N., M.P.G., D.D.W., J.G.Z., S.K., J.H., and J.S. All authors were involved in the decision to submit the manuscript for publication.

This project would not have been possible without the support of the AMPATH and members of the IU-Kenya partnership. Many individuals contributed to the implementation of the clinic-based survey under the direction of the authors. The authors acknowledge the contributions of interviewer and data entry teams, as well as the CRHC staff under the direction of Dr Moses Kigani.

The views expressed here do not necessarily reflect those of the World Bank or its member countries, the United States Agency for International Development or the United States Government. 
sent at a daily or weekly frequency. Adherence was measured using the medication event monitoring system. The primary outcome was whether adherence exceeded $90 \%$ during each 12 week period of analysis and the 48-week study period. The secondary outcome was whether there were treatment interruptions lasting at least $48 \mathrm{~h}$.

Results-In intention-to-treat analysis, 53\% of participants receiving weekly SMS reminders achieved adherence of at least $90 \%$ during the 48 weeks of the study, compared with $40 \%$ of participants in the control group $(P=0.03)$. Participants in groups receiving weekly reminders were also significantly less likely to experience treatment interruptions exceeding $48 \mathrm{~h}$ during the 48 week follow-up period than participants in the control group ( 81 vs. $90 \%, P=0.03)$.

Conclusion-These results suggest that SMS reminders may be an important tool to achieve optimal treatment response in resource-limited settings.

\section{Keywords}

adherence; antiretroviral therapy; HIV/AIDS; mobile phones; randomized controlled trial; subSaharan Africa; text message reminders

\section{Introduction}

Although antiretroviral therapy (ART) has dramatically reduced morbidity and mortality for people living with HIV/AIDS, incomplete treatment adherence is the major cause of treatment failure, development of drug resistance, HIV disease progression, and death [1-5]. Initial concerns about poor adherence and widespread drug resistance in resource-limited settings have not been realized [6-8]. Many studies indicate that adherence in resourcelimited settings is at least as good, if not better than in North America [9-12]. However, evidence from large-scale treatment programs has been lacking, and some studies have found that adherence among individuals in sub-Saharan Africa declines over time [13,14]. Given the prohibitive cost of second-line therapy, successful adherence support interventions may be cost saving [11,12].

There is no consensus about the best approach to improve adherence in resource-limited settings [15]. Significant challenges include the large numbers of individuals on ART who are residentially dispersed and who face structural and economic constraints to sustained treatment access [9]. However, many resource-limited settings have well developed cellular telecommunication networks and mobile phone ownership worldwide has grown dramatically from 1 billion in 2002 to 4.1 billion in 2008 [16]. Mobile phones have been shown to improve chronic disease management in developed countries and have been proposed as a potential strategy to support ART adherence in developing countries [17-19]. Reminder devices, however, have shown mixed results and few data are available for resource-limited settings [20-22]. Encouragingly, a study conducted in Mombasa, Kenya, found that alarm devices significantly improved non-ART medication adherence rates among women attending sexually transmitted disease and family planning clinics [23].

\section{Methods}

\section{Participants and setting}

The study was conducted at the Chulaimbo Rural Health Center (CRHC) in Nyanza Province, Kenya. CRHC is a government-run health facility that has hosted an HIV clinic run by the Academic Model Providing Access to Healthcare (AMPATH) since 2005. At the time of the study, $45 \%$ of study participant households reported cell phone ownership and 97\% resided within cell phone network coverage. 
All participants gave informed consent to participation in the study, which was approved by the Institutional Research Ethics Committee of Moi University and the human subjects committee of Georgetown University. Study participants were recruited at AMPATH's HIV clinic at CRHC. Recruitment began on 17 June 2007. Patients older than 18 years of age who had initiated ART less than 3 months prior to enrollment were eligible for the study. Eligible patients were informed about the study and then asked for consent in one of three languages (English, Dholuo, and Kiswahili). All participants were informed that they would receive a mobile phone and that some would be randomly selected to receive daily or weekly text messages encouraging adherence to ART. They were also informed that one of their medications would be dispensed in bottles with electronic caps monitoring daily usage.

\section{Interventions and study design}

Participants were provided with a Nokia mobile phone and told that they could use it as they desired. The study did not restrict enrollment to participants who already owned a phone to avoid selection biases from preexisting phone ownership. Participants were referred to the pharmacy, wherein one of their three antiretroviral medications (in all but one case lamivudine) was transferred to a bottle with a medication event monitoring system (MEMS) cap by the pharmacy staff. The phone number and MEMS cap numbers of participants were recorded by study staff.

Participants were randomly assigned to one of four intervention groups or to the control group that would receive no text messages. One-third of the sample was allocated to the control group, and the remaining two-thirds of the sample were allocated evenly to each of the four intervention groups. The randomization schedule was prepared in advance of enrollment by the investigators. A sequence of random numbers between 0 and 1 were generated, and four equal intervals between 0 and $2 / 3$ corresponded to the four intervention groups, whereas the value interval from $2 / 3$ to 1 corresponded to the control group.

The four text message interventions were chosen to address different barriers to adherence such as forgetfulness and lack of social support [24,25]. Short messages were meant to serve as a simple reminder to take medications, whereas long messages were meant to provide additional support. Daily messages were close to the frequency of medication usage, whereas weekly messages were meant to avoid the possibility that very frequent text messages would be habituating. The content of each type of message is listed in Table 1.

The message content was developed after extensive consultation with clinic staff. Correct phone use and comprehension of the message in English, Dholou, or Kiswahili was confirmed during a basic 15-min training session. All messages were less than 160 characters and did not specify HIV or ART in order to maintain confidentiality of HIV status. Participants were asked to specify their preferred language. Messages were sent automatically initially by a commercial service provider (Zunguka) based in Nairobi and later from Cardboard-Fish.com. The service sent 'one-way' messages to which respondents could not respond. Messages were sent at 12 p.m., rather than twice daily (during dosing times) to avoid excess reliance on the accuracy of the short message service (SMS) software.

\section{Follow-up}

Participants were expected to return to the clinic once a month as per AMPATH's standard procedures. MEMS caps were scanned monthly by study staff in the pharmacy.

The study provided regular assistance to participants in the intervention and control groups owing to poor access to electricity and household financial constraints. As many participants needed to charge their phones at fee-based 'charging stations' in nearby market centers, the study provided 80 Kenya Shillings (approximately 1 US\$) at every monthly visit. In 
addition, as the subscriber identity module (SIM) cards in phones would expire if phone credit was not added regularly, 50 Kenya Shillings of phone credit was added to participants' phones every 2 months.

Participants were required to show their study-provided phone to the study staff during their clinic visit. Participants who reported having lost their phones were not provided with a replacement phone. To confirm the fidelity of the interventions, each of the messages were sent on a daily or weekly basis to a separate phone maintained by the study supervisor to ensure server functioning. Functionality of each participant's phone was checked during each visit.

\section{Sample sizes}

The study was designed to have $90 \%$ power to detect an impact of $15 \%$ in the fraction of patients with adherence of at least $90 \%$ (assuming a no intervention median adherence level of $90 \%$ ), using a two-tailed test of significance at the $5 \%$ level.

The study population consisted of all participants enrolled between June 2007 and August 2008. SMS reminders were sent to intervention groups until 31 December 2008. We restricted analyses to participants who were enrolled before 31 January 2008, the date by which a 48-week follow-up was possible.

\section{Medication event monitoring system use and data management}

Several adjustments were made to the MEMS data to address variation in regimen and MEMS cap use. First, refill MEMS events were censored. Second, many patients experienced a clinic-driven regimen change to either fixed-dose combination of zidovudine/ lamivudine or fixed-dose combination of stavudine/lamivudine/ nevirapine during the first half of 2008. These participants received the new medication in the MEMS bottle, but were instructed to complete existing doses, which were transferred to an envelope. The number of days of the remaining medication was recorded and censored.

MEMS adherence was calculated as the number of actual bottle openings divided by the number of prescribed bottle openings for the period. Because all patients were prescribed twice-daily ART, we set the maximum number of daily openings equal to two so that MEMS adherence was not inflated by extra cap openings. Our primary outcome was a binary indicator of whether the patient had adherence of at least $90 \%$ during each 12 -week period of analysis. As treatment interruptions are an important predictor of virologic failure on nonnucleoside reverse transcriptase inhibitor therapy, our secondary outcome was a binary indicator of whether patients had a treatment interruption exceeding $48 \mathrm{~h}$ during each period of analysis [26]. An interruption was defined as having occurred if the time between two consecutive MEMS openings exceeded $48 \mathrm{~h}$.

\section{Statistical analysis}

We conducted an 'inside the table' analysis of our factorial research design. Differences in the primary and secondary outcomes between each of the intervention groups and the control group were compared using the $\chi^{2}$-test for each 12-week period of observation (1$12,13-24,25-36$, and 37-48 weeks), as well as the full 48-week follow-up period. Two levels of analysis were conducted: a 'summary analysis' in which we compared the effects of the daily, weekly, short and long reminders to the control group and a 'subgroup analysis' in which we compared each of the four intervention arms to the control group.

Our primary analysis was performed on an intention-to-treat basis. Individuals who discontinued therapy or who were lost to follow-up were classified as nonadherent for the 
relevant observation period; they were coded as not having achieved adherence of at least $90 \%$ and as having experienced a treatment interruption exceeding $48 \mathrm{~h}$. Our secondary analysis was per-protocol, which compared adherence for participants retained in care for each 12-week period of enrollment in the study and over the entire 48 weeks. In this analysis, participants lost to follow-up during a given period were not included in the analysis for that period. All analyses were conducted with STATA version 10.0 (StataCorp, College Station, Texas, USA).

\section{Results}

\section{Characteristics of the study population}

Seven hundred and thirty-five patients were approached and 720 (97.9\%) were enrolled (Fig. 1). Our analyses are restricted to the 431 participants who were enrolled before 31 January 2008. A total of three participants had faulty MEMS caps and were excluded from the analysis because of failure to reconstruct their MEMS data, resulting in an analytical sample of 428 participants.

Among participants included in the analyses, 139 were in the control group, 70 in the short daily reminders group, 72 in the long daily reminders group, 73 in the short weekly reminders group, and 74 in the long weekly reminders group. The most common antiretroviral medications provided at enrollment were lamivudine (99.8\%), nevirapine (88\%), and zidovudine (64\%). There is no difference across the control and each of the intervention groups in the likelihood of switching regimens during the study $\chi^{2}$-test, $P=0.76)$.

Table 2 shows that at baseline, most participants' characteristics such as age, sex, religion, ethnicity, marital status, and education were distributed similarly across the five groups in the analytical sample. The only characteristic in -which there were significant differences between groups -was residence in a home -with an iron roof $(P=0.04)$. In addition, Table 2 shows that the analytical sample of 428 study participants -was not significantly different from the entire sample of 720 participants -who -were enrolled in the study.

\section{Retention of participants}

Participants in the study were defined as lost to follow-up if more than 90 days had elapsed after their last recorded MEMS opening. Sixty-nine participants, or $16 \%$ of the original sample, were lost to follow-up as of 31 December 2008. This is consistent -with typical loss to follow-up rates in similar programs [27,28]. The percentage of participants lost to followup by group -was as follows: control group (14.4\%), short daily reminders group (18.6\%), long daily reminders group (16.7\%), short weekly reminders group (22\%), and long -weekly reminders group (10.8\%). There was no significant difference in the rate of loss to follow-up across the control and each of the four intervention groups $(P=0.48)$.

\section{Fidelity of intervention}

A total of 69 participants lost their phones and 51 changed phone numbers during the course of the study (this was usually due to patients' opting to use their original SIM card and phone number). The likelihood of losing the phone or changing phone numbers was not significantly different across five groups (lost phones, $\chi^{2}$-test, $P=0.60$; changed number, $\chi^{2}$-test, $P=0.62$ ). Twenty-five participants with a malfunctioning MEMS caps or with a lost MEMS cap were provided with a replacement cap for their medications. There were no differences in the likelihood of MEMS cap replacement ( $\chi^{2}$-test, $P=0.59$ ). 


\section{Adherence in the control group}

Overall adherence for participants in the control group retained in care for 48 weeks was $75.8 \%$ and declined over the follow-up period. Table 3 shows that the fraction of participants in the control group achieving adherence of at least $90 \%$ was $60 \%$ in weeks 1 12 and declined to $46 \%$ in weeks $37-48\left(\chi^{2}=7.36, P=0.007\right)$. In addition, $90 \%$ of the control group experienced at least one treatment interruption exceeding $48 \mathrm{~h}$ (Table 5). The percentage of participants with treatment interruptions also rose over time, from $40 \%$ in weeks $1-12$ to $58 \%$ in weeks $37-48(P=0.001)$.

\section{Intervention effects on at least $90 \%$ adherence}

Intention-to-treat analysis (daily and weekly reminders)-Table 3 describes the percentage of participants achieving adherence of at least $90 \%$ for each group in weeks 1 $12,13-24,25-36,37-48$, and $0-48$. The top panel shows the summary results, whereas the bottom panel shows the four intervention groups' results. The fraction of participants with adherence of at least $90 \%$ in the two groups receiving weekly reminders was significantly higher than the control group (53 vs. $40 \%, P=0.03$ ). The fraction of participants with adherence of at least $90 \%$ was not significantly greater for those receiving daily reminders than the control group (41 vs. $40 \%, P=0.92$ ).

Intention-to-treat analysis (short and long reminders)—Adherence of participants in the two groups receiving short or long reminders was similar to the control group in all four quarters of the study. Over 48 weeks, the fraction of participants achieving adherence of at least $90 \%$ was $47 \%$ in the two groups that received long reminders, compared with $40 \%$ in the control group $(P=0.24)$. Similarly, the two groups receiving short reminders achieved adherence of at least $90 \%$ at rates that were not significantly higher than the control group ( 47 vs. $40 \%, P=0.24$ ).

Per-protocol analysis (daily and weekly reminders)_Among participants who were retained in care at 48 weeks, the fraction who achieved at least $90 \%$ adherence was $63 \%$ for the group that received weekly reminders (Table 4 ). This is significantly higher than the control group (63 vs. $47 \%, P=0.01$ ). The subgroup analysis suggests that this result is largely driven by the group receiving short weekly reminders and the control group (68 vs. $47 \%, P=0.01$ ). However, we acknowledge that lower retention in this group may have impacted the adherence estimate. On the contrary, the group receiving long weekly reminders was not significantly more adherent than the control group $(P=0.12)$. Participants in the intervention groups receiving daily messages were not more likely to be adherent (50 vs. $47 \%, P=0.70)$.

Per-protocol analysis (short and long reminders)-The two groups receiving short reminders and retained in care for 48 weeks were marginally more likely to be adherent than the control group (59 vs. $47 \%, P=0.07$ ). The proportion achieving adherence of at least $90 \%$ in the two groups receiving long reminders and retained in care in each observation period was similar to the control group (55 vs. $47 \%, P=0.23$ ).

\section{Intervention effects on at least $48 \mathrm{~h}$ treatment interruptions}

Intention-to-treat analysis (daily and weekly reminders)-Table 5 shows the percentage of participants with at least one treatment interruption exceeding $48 \mathrm{~h}$ in each 12 week period and over the entire 48 weeks. Participants lost to follow-up are classified as having experienced a treatment interruption exceeding $48 \mathrm{~h}$ in this analysis. Participants in the groups receiving weekly reminders were significantly less likely to experience at least one treatment interruption over the entire follow-up period than participants in the control 
group ( 81 vs. $90 \%, P=0.03$ ). Participants receiving the daily messages were not significantly less likely to report at least one treatment interruption greater than $48 \mathrm{~h}$ (86 vs. $90 \%, P=0.30$ ).

Intention-to-treat analysis (short and long reminders)-Participants receiving the long message were marginally less likely to report at least one interruption exceeding $48 \mathrm{~h}$ over the 48 -week period ( 83 vs. $90 \%, P=0.08$ ). Participants receiving the short message were as likely to report a treatment interruption as the controls group ( 84 vs. $90 \%, P=0.14$ ).

Per-protocol analysis (daily and weekly reminders) - In Table 6, the fraction of individuals with at least one treatment interruption exceeding $48 \mathrm{~h}$ and retained in care for 48 weeks in the two groups receiving weekly messages was significantly lower than the control group ( 77 vs. $88 \%, P=0.02$ ). The two groups receiving daily messages were as likely as the control group to report a treatment interruption over 48 weeks ( 83 vs. $88 \%, P=0.24$ ).

Per-protocol analysis (short and long reminders)—Participants retained in care at the end of the 48-week study period and receiving either the short or long messages were slightly less likely to report at least one treatment interruption over the 48 -week period than the control group ( $80 \mathrm{vs.} 88 \%, P=0.08$ ). The subgroup analysis suggests that this result is driven by the intervention group that received weekly messages.

\section{Discussion}

Our data indicate that weekly SMS reminders increased the percentage of participants achieving $90 \%$ adherence to ART by approximately $13-16 \%$ compared with no reminder. These weekly reminders were also effective at reducing the frequency of treatment interruptions, which have been shown to be an important cause of treatment resistant failure in resource-limited settings [29]. Like other successful adherence interventions, the intervention was effective in part by preventing the decline in adherence seen in the control group from 60 to $46 \%$ achieving 90\% adherence over 48 weeks [21,30,31]. Despite SMS outages, phone loss, and a rural population, these results suggest that simple SMS interventions could be an important strategy to sustaining optimal ART response. Preventing adherence-related treatment failure is especially important in resource-limited settings wherein second-line therapy is up to 17 -fold more expensive than first-line therapy and often unavailable [32].

Contrary to our expectations, adding words of encouragement in the longer text message reminders was not more effective than either a short reminder or no reminder. Although previous studies have shown benefit with individualized adherence tools [33,34], future cell phone-based interventions should investigate how message form and content influence HIV and other chronic disease-related behavior [35,36]. Message content must also take into account the common practice of shared mobile phone use and the potential that individuals will change phones numbers [37].

It is also interesting to note that weekly reminders improved adherence, whereas daily reminders did not. Habituation, or the diminishing of a response to a frequently repeated stimulus, may explain this finding. Daily messages might also have been considered intrusive. Further research is needed to distinguish the mechanisms as to why the weekly messages were most efficacious. Moreover, this study was not designed to link the sending of a reminder with the actual time the individual opened the MEMS bottle. Wireless technology has recently enabled real-time adherence monitoring, such that reminders can be explicitly linked to missed dosages [38]. 
The level of adherence we observed was less than what has been seen in early reports using electronic monitoring [39]. This finding may reflect the fact that we censored all openings in excess of two openings per day, which can lead to a falsely lower adherence estimate when individuals take their evening dose just after midnight on the following day. Alternatively, it is possible that population levels of adherence may be declining in rural resource-limited settings as treatment access expands and there is less selection towards highly adherent individuals starting ART [40,41].

There are several limitations to our study. We cannot positively distinguish whether the intervention improved dose-taking behavior or simply improved use of the electronic medication monitor. Individuals may remove several doses of ART from the MEMS bottle at one time (also known as 'pocket doses') or simply cease using the monitor [42]. However, there is no strong reason to believe that 'pocket doses' were taken at different rates by intervention and control groups. Another limitation is that we do not have HIV-RNA determinations and, therefore, cannot corroborate that the differences in adherence were associated with differences in viral suppression. Finally, we measured adherence in only one tablet and assume that this reflects adherence of the entire regimen.

The results of this study provide promising evidence that automated text message reminders may improve adherence among patients initiating ART in resource-limited settings. This strategy could be used for wide-scale improvement of adherence given the convenience and low cost of delivering SMS. A single server can provide text messages to thousands of patients over a wide geographic area and few human resources are needed beyond the initial setup. This strategy could be a key component of comprehensive ART adherence support [16]. However, due to the customary use of mobile phones by more than one individual in some settings, cell phone interventions will need to continue to address issues of confidentiality.

This study is among the first to present robust evidence of beneficial effects of mobile phone technology for HIV/ AIDS care delivery, also known as mHealth. Although the potential for mobile phones to make an impact on health has been popularized in the literature and lay press, little evidence had been provided to support such claims [43,44]. Research is needed to determine the reproducibility and generalizability of these findings. Moreover, beyond adherence reminders, mobile phones may be used for appointment reminders, monitoring of adherence and treatment side-effects, and other types of communication between patients and healthcare workers in between clinic visits. Additional studies will be critical for understanding the true benefits and best implementation strategies for mHealth in developing countries.

\section{Acknowledgments}

The World Bank Research Group provided financial support for this study under contracts 7142349 and 7144565 funded by the Bank Netherlands Partnership Program (BNPP). This research was also supported in part by a grant to the USAID-AMPATH Partnership from the United States Agency for International Development as part of the President's Emergency Plan for AIDS Relief (PEPFAR). J.H. and D.R.B. received support from the National Institute of Mental Health (87228 and 87227, respectively).

\section{References}

1. Palella FJ Jr. Delaney KM, Moorman AC, Loveless MO, Fuhrer J, Satten GA, et al. Declining morbidity and mortality among patients with advanced human immunodeficiency virus infection. HIV Outpatient Study Investigators. N Engl J Med. 1998; 338:853-860. [PubMed: 9516219]

2. Hogg RS, Heath K, Bangsberg D, Yip B, Press N, O'Shaughnessy MV, et al. Intermittent use of triple-combination therapy is predictive of mortality at baseline and after 1 year of follow-up. AIDS. 2002; 16:1051-1058. [PubMed: 11953472] 
3. Wood E, Hogg RS, Yip B, Harrigan PR, O'Shaughnessy MV, Montaner JS. The impact of adherence on CD4 cell count responses among HIV-infected patients. J Acquir Immune Defic Syndr. 2004; 35:261-268. [PubMed: 15076240]

4. Bangsberg DR, Perry S, Charlebois ED, Clark RA, Roberston M, Zolopa AR, Moss A. Nonadherence to highly active antiretroviral therapy predicts progression to AIDS. AIDS. 2001; 15:1181-1183. [PubMed: 11416722]

5. Hogg R, Heath K, Bangsberg D, Yip B, Press N, O’Shaughnessy MV, Montaner JS. Intermittent use of triple-combination therapy is predictive of mortality at baseline and after 1 year of follow-up. AIDS. 2002; 16:1051-1058. [PubMed: 11953472]

6. Bartlett JA, Shao JF. Successes, challenges, and limitations of current antiretroviral therapy in lowincome and middle-income countries. Lancet Infect Dis. 2009; 9:637-649. [PubMed: 19778766]

7. Harries AD, Nyangulu DS, Hargreaves NJ, Kaluwa O, Salaniponi FM. Preventing antiretroviral anarchy in sub-Saharan Africa. Lancet. 2001; 358:410-414. [PubMed: 11502341]

8. Gill CJ, Hamer DH, Simon JL, Thea DM, Sabin LL. No room for complacency about adherence to antiretroviral therapy in sub-Saharan Africa. AIDS. 2005; 19:1243-1249. [PubMed: 16052078]

9. Mills EJ, Nachega JB, Buchan I, Orbinski J, Attaran A, Singh S, et al. Adherence to antiretroviral therapy in sub-Saharan Africa and North America: a meta-analysis. JAMA. 2006; 296:679-690. [PubMed: 16896111]

10. Orrell C, Bangsberg DR, Badri M, Wood R. Adherence is not a barrier to successful antiretroviral therapy in South Africa. AIDS. 2003; 17:1369-1375. [PubMed: 12799558]

11. Nachega JB, Hislop M, Nguyen H, Dowdy DW, Chaisson RE, Regensberg L, et al. Antiretroviral therapy adherence, virologic and immunologic outcomes in adolescents compared with adults in southern Africa. J Acquir Immune Defic Syndr. 2009; 51:65-71. [PubMed: 19282780]

12. Unge C, Sodergard B, Ekstrom AM, Carter J, Waweru M, llako F, et al. Challenges for scaling up ART in a resource-limited setting: a retrospective study in Kibera, Kenya. J Acquir Immune Defic Syndr. 2009; 50:397-402. [PubMed: 19214119]

13. Byakika-Tusiime J, Crane J, Oyugi JH, Ragland K, Kawuma A, Musoke P, Bangsberg DR. Longitudinal antiretroviral adherence in HIV+ Ugandan parents and their children initiating HAART in the MTCT-plus family treatment model: role of depression in declining adherence over time. AIDS Behav. 2009; 13(Suppl 1):82-91. [PubMed: 19301113]

14. Mannheimer SB, Matts J, Telzak E, Chesney M, Child C, Wu AW, Friedland G. Terry Beirn Community Programs for Clinical Research on AIDS. Quality of life in HIV-infected individuals receiving antiretroviral therapy is related to adherence. AIDS Care. 2005; 17:10-22. [PubMed: 15832830]

15. Simoni JM, Pearson CR, Pantalone DW, Marks G, Crepaz N. Efficacy of interventions in improving highly active antiretroviral therapy adherence and HIV-1 RNA viral load. A metaanalytic review of randomized controlled trials. J Acquir Immune Defic Syndr. 2006; 43(Suppl 1):S23-S35. [PubMed: 17133201]

16. International Telecommunications Union. Measuring the information society: the ICT development index. Geneva, Switzerland: International Telecommunication Union; 2009.

17. Fjeldsoe BS, Marshall AL, Miller YD. Behavior change interventions delivered by mobile telephone short-message service. Am J Prev Med. 2009; 36:165-173. [PubMed: 19135907]

18. Chang LW, Kagaayi J, Nakigozi G, Packer AH, Serwadda D, Qui nn TC, et al. Responding to the human resource crisis: peer health workers, mobile phones, and HIV care in Rakai, Uganda. AIDS Patient Care STDS. 2008; 22:173-174. [PubMed: 18290750]

19. Lester R, Karanja S. Mobile phones: exceptional tools for HIV/AIDS, health, and crisis management. Lancet Infect Dis. 2008; 8:738-739. [PubMed: 19022188]

20. Andrade AS, McGruder HF, Wu AW, Celano SA, Skolasky RL Jr. Selnes OA, et al. A programmable prompting device improves adherence to highly active antiretroviral therapy in HIV-infected subjects with memory impairment. Clin Infect Dis. 2005; 41:875-882. [PubMed: 16107989]

21. Mannheimer SB, Morse E, Matts JP, Andrews L, Child C, Schmetter B, Friedland GH. Terry Beirn Community Programs for Clinical Research on AIDS. Sustained benefit from a long-term 
antiretroviral adherence intervention. Results of a large randomized clinical trial. J Acquir Immune Defic Syndr. 2006; 43(Suppl 1):S41-S47. [PubMed: 17091022]

22. Wise J, Operario D. Use of electronic reminder devices to improve adherence to antiretroviral therapy: a systematic review. AIDS Patient Care STDS. 2008; 22:495-504. [PubMed: 18462071]

23. Frick PA, Lavreys L, Mandaliya K, Kreiss JK. Impact of an alarm device on medication compliance in women in Mombasa, Kenya. Int J STD AIDS. 2001; 12:329-333. [PubMed: 11368808]

24. Castro A. Adherence to antiretroviral therapy: merging the clinical and social course of AIDS. PLoS Med. 2005; 2:e338. [PubMed: 16187735]

25. Mills EJ, Nachega JB, Bangsberg DR, Singh S, Rachlis B, Wu P, et al. Adherence to HAART: a systematic review of developed and developing nation patient-reported barriers and facilitators. PLoS Med. 2006; 3:e438. [PubMed: 17121449]

26. Parienti JJ, Das-Douglas M, Massari V, Guzman D, Deeks SG, Verdon R, Bangsberg DR. Not all missed doses are the same: sustained NNRTI treatment interruptions predict HIV rebound at lowto-moderate adherence levels. PLoS One. 2008; 3:e2783. [PubMed: 18665246]

27. Wools-Kaloustian K, Kimaiyo S, Diero L, Siika A, Sidle J, Yiannoutsos CT, et al. Viability and effectiveness of large-scale HIV treatment initiatives in sub-Saharan Africa: experience from western Kenya. AIDS. 2006; 20:41-48. [PubMed: 16327318]

28. Rosen S, Fox MP, Gill CJ. Patient retention in antiretroviral therapy programs in sub-Saharan Africa: a systematic review. PLoS Med. 2007; 4:e298. [PubMed: 17941716]

29. Oyugi JH, Byakika-Tusiime J, Ragland K, Laeyendecker O, Mugerwa R, Kityo C, et al. Treatment interruptions predict resistance in HIV-positive individuals purchasing fixed-dose combination antiretroviral therapy in Kampala, Uganda. AIDS. 2007; 21:965-971. [PubMed: 17457090]

30. Remien RH, Stirratt MJ, Dolezal C, Dognin JS, Wagner GJ, Carballo-Dieguez A, et al. Couplefocused support to improve HIV medication adherence: a randomized controlled trial. AIDS. 2005; 19:807-814. [PubMed: 15867495]

31. Liu H, Miller LG, Hays RD, Golin CE, Wu T, Wenger NS, Kaplan AH. Repeated measures longitudinal analyses of HIV virologic response as a function of percentage adherence, dose timing, genotypic sensitivity, and other factors. J Acquir Immune Defic Syndr. 2006; 41:315-322. [PubMed: 16540932]

32. Medicins Sans Frontiers. Untangling the web of antiretroviral therapy price reductions. Geneva, Switzerland: Medicins Sans Frontiers; 2010.

33. Safren SA, Hendriksen ES, Desousa N, Boswell SL, Mayer KH. Use of an on-line pager system to increase adherence to antiretroviral medications. AIDS Care. 2003; 15:787-793. [PubMed: 14617500]

34. Ostrop NJ, Gill MJ. Antiretroviral medication adherence and persistence with respect to adherence tool usage. AIDS Patient Care STDS. 2000; 14:351-358. [PubMed: 10935052]

35. Fisher JD, Cornman DH, Norton WE, Fisher WA. Involving behavioral scientists, healthcare providers, and HIV-infected patients as collaborators in theory-based HIV prevention and antiretroviral adherence interventions. J Acquir Immune Defic Syndr. 2006; 43(Suppl 1):S10-S17. [PubMed: 17133191]

36. Elder JP, Ayala GX, Harris S. Theories and intervention approaches to health-behavior change in primary care. Am J Prev Med. 1999; 17:275-284. [PubMed: 10606196]

37. Shet A, Arumugam K, Rodrigues R, Rajagopalan N, Shubha K, Raj T, et al. Designing a mobile phone-based intervention to promote adherence to antiretroviral therapy in south India. AIDS Behav. 2010; 4:716-720. [PubMed: 20054634]

38. Bangsberg DR, Deeks SG. Spending more to save more: interventions to promote adherence. AnnIntern Med. 2010; 152:54-56. W-13.

39. Oyugi JH, Byakika-Tusiime J, Charlebois ED, Kityo C, Mugerwa R, Mugyenyi P, Bangsberg DR. Multiple validated measures of adherence indicate high levels of adherence to generic HIV antiretroviral therapy in a resource-limited setting. J Acquir Immune Defic Syndr. 2004; 36:11001102. [PubMed: 15247564] 
40. Vriesendorp R, Cohen A, Kristanto P, Vrijens B, Rakesh P, Anand B, et al. Adherence to HAART therapy measured by electronic monitoring in newly diagnosed HIV patients in Botswana. Eur J Clin Pharmacol. 2007; 63:1115-1121. [PubMed: 17882408]

41. Bell DJ, Kapitao Y, Sikwese R, van Oosterhout JJ, Lalloo DG. Adherence to antiretroviral therapy in patients receiving free treatment from a government hospital in Blantyre, Malawi. J Acquir Immune Defic Syndr. 2007; 45:560-563. [PubMed: 17558333]

42. Deschamps AE, Graeve VD, van Wijngaerden E, De Saar V, Vandamme AM, van Vaerenbergh K, et al. Prevalence and correlates of nonadherence to antiretroviral therapy in a population of HIV patients using medication event monitoring system. AIDS Patient Care STDS. 2004; 18:644-657. [PubMed: 15633262]

43. The Economist. Beyond voice: new uses for mobile phones could launch another wave of development. The Economist Newspaper Limited. 2009 Sep 24.

44. Kaplan WA. Can the ubiquitous power of mobile phones be used to improve health outcomes in developing countries? Global Health. 2006; 2:9. [PubMed: 16719925] 


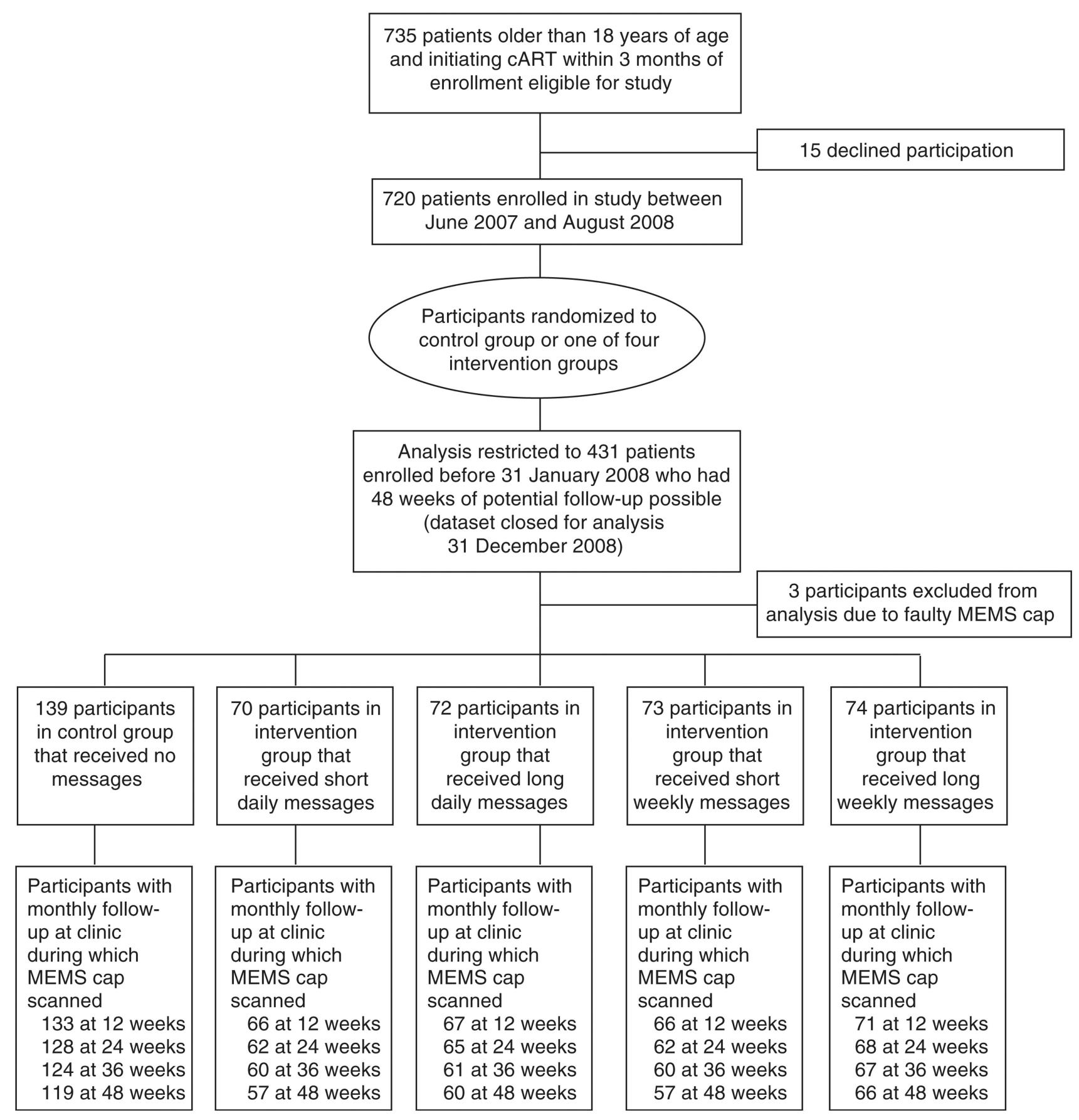

Fig. 1. Flowchart of randomized controlled trial

cART, combination antiretroviral therapy; MEMS, medication event monitoring system. 


\section{Table 1}

Content of short and long short message service reminders.

\begin{tabular}{llll}
\hline & English & Swahili & Dholuo \\
\hline Short reminder & This is your reminder. & Hili ni kumbukumbu lako. & Ma en ote ma iparonigo. \\
Long reminder & $\begin{array}{l}\text { This is your reminder. Be strong } \\
\text { and courageous, we care about you. }\end{array}$ & $\begin{array}{l}\text { Hili ni kumbukumbu lako. } \\
\text { Uwe na ujasiri, tunakujali. }\end{array}$ & $\begin{array}{l}\text { Ma en ote ma iparonigo. Bed motegno } \\
\text { kendo bed gi chir, wageni. }\end{array}$ \\
\hline
\end{tabular}




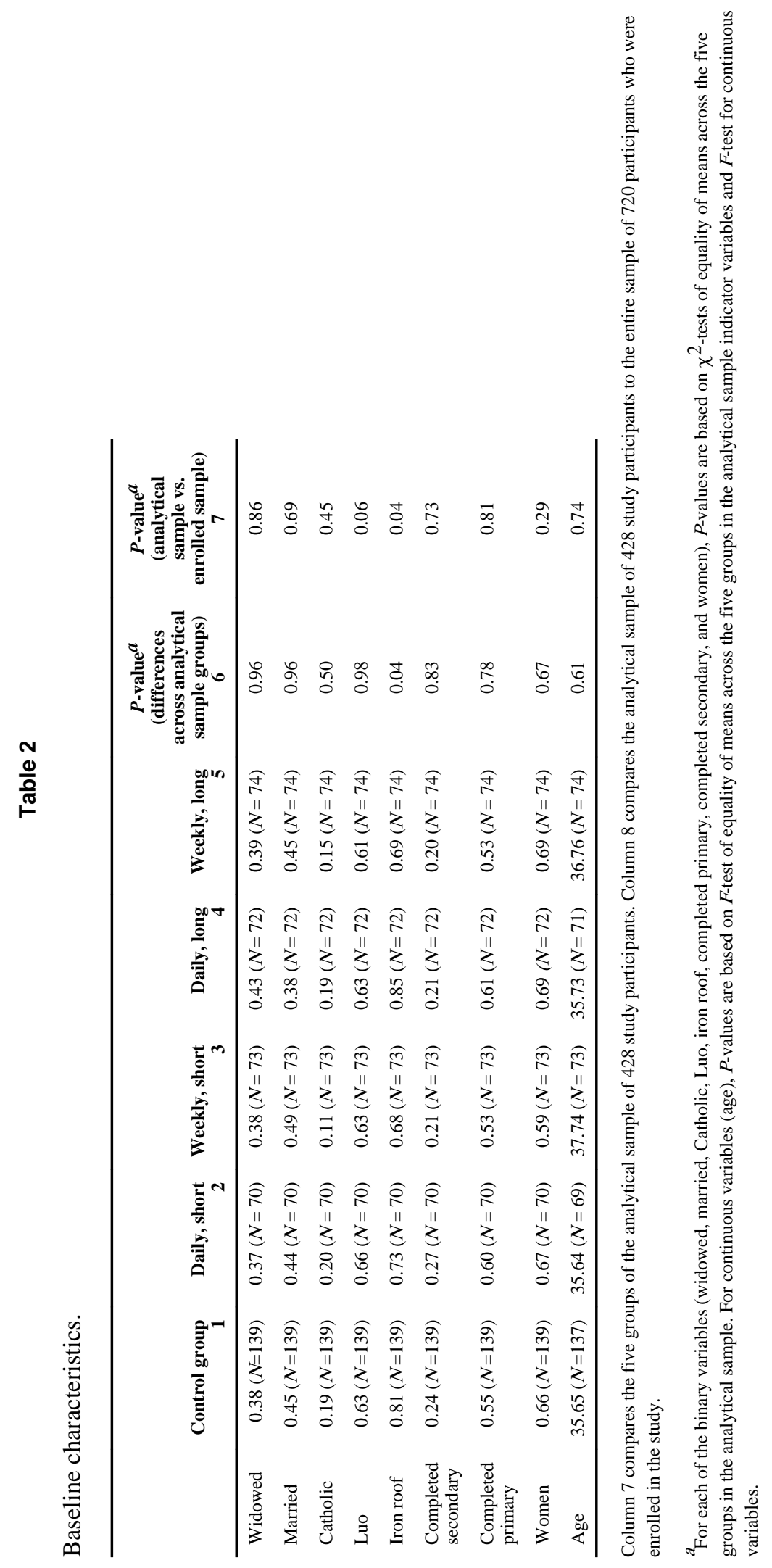




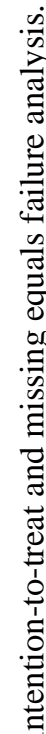

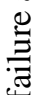

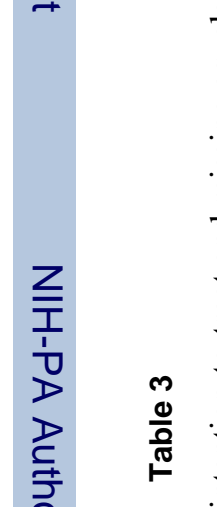




$$
\begin{aligned}
& \stackrel{+}{\frac{0}{\circ}}
\end{aligned}
$$

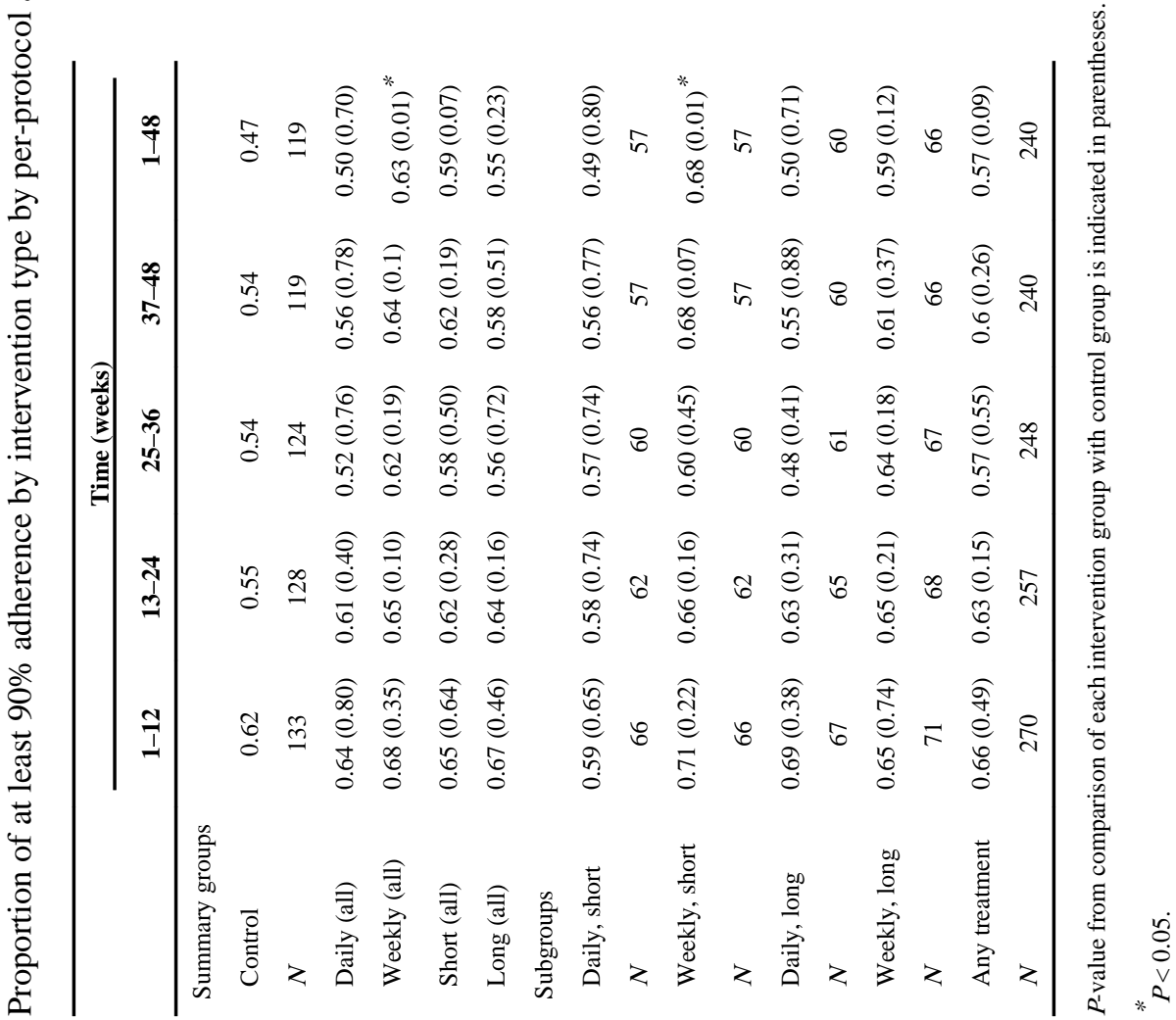


0
$\frac{1}{0}$
$\frac{0}{10}$ 